\section{$\underset{\substack{\text { hommes } \\ \text { \& migrations }}}{ }$}

\section{Hommes \& migrations}

Revue française de référence sur les dynamiques

migratoires

1302 | 2013

Le Japon, pays d'immigration?

\title{
Les élèves étrangers au Japon
}

Un accès inégal à l'éducation

\section{Daïsuké Sonoyama}

\section{(2) OpenEdition}

\section{Journals}

Édition électronique

URL : http://journals.openedition.org/hommesmigrations/2459

DOI : 10.4000/hommesmigrations.2459

ISSN : 2262-3353

Éditeur

Musée national de l'histoire de l'immigration

Édition imprimée

Date de publication : 1 avril 2013

Pagination : $57-64$

ISBN : 978-2-919040-22-3

ISSN : $1142-852 X$

\section{Référence électronique}

Daïsuké Sonoyama, "Les élèves étrangers au Japon », Hommes \& migrations [En ligne], 1302 | 2013, mis en ligne le 01 avril 2016, consulté le 10 décembre 2020. URL : http://journals.openedition.org/ hommesmigrations/2459; DOI : https://doi.org/10.4000/hommesmigrations.2459 


\title{
LES ÉLĖVES ÉTRANGERS AU JAPON UN ACCÈS INÉGAL À L'ÉDUCATION
}

par DAÏSUKÉ SONOYAMA, maître de conférences en sciences de l'éducation, université d'Osaka ${ }^{1}$

\author{
Maîtriser la langue japonaise est la condition nécessaire \\ pour suivre l'enseignement public au Japon. Dans un système \\ conçu pour les Japonais, les élèves étrangers ont un besoin \\ crucial de soutien linguistique. Partagée entre l'État et \\ les collectivités locales, la politique éducative peine à s'adapter \\ à la diversité de la société japonaise. Des écoles ethniques \\ existent, mais elles bénéficient d'une reconnaissance aléatoire \\ et n'ouvrent pas toujours les portes de l'enseignement \\ supérieur universitaire. Ainsi, l'éducation devient un instrument \\ de discrimination.
}

\section{Un système éducatif fondé sur l'assimilation}

La politique éducative vis-à-vis des élèves étrangers au Japon est aujourd'hui au centre des débats. Quelques études historiques et sociologiques menées par des chercheurs japonais ont déjà souligné les situations d'inégalité scolaire au Japon et se sont prononcées en faveur d'une politique de discrimination positive pour offrir aux étrangers un meilleur accès à l'éducation². Malheureusement, la Constitution du Japon ainsi que la loi fondamentale sur l'éducation discriminent les citoyens non japonais. Des efforts sont faits par les collectivités territoriales, les chefs d'établissement ou les enseignants qui s'engagent en faveur de l'accueil des étrangers avec le soutien des parents ou d'autres groupes de bénévoles pour l'intégration des élèves d'origine étrangère. Mais ces initiatives ne permettent toujours pas d'assurer un accès égal pour tous à l'enseignement et une équité au sein du système scolaire.

Le présent article repose sur l'analyse des lois relatives à l'éducation, des mesures politiques prises aux niveaux local et national depuis les années 1970, ainsi que sur les statistiques et les enquêtes fournies par les ministères de l'Éducation, de la Justice, des Affaires intérieures et des Communications. 


\section{La discrimination des élèves étrangers et les problèmes de soutien linguistique}

Il est nécessaire de donner, tout d'abord, quelques données statistiques pour faire un état des lieux du problème des élèves étrangers dans l'enseignement scolaire au Japon. Le nombre total d'élèves étrangers inscrits dans les écoles étrangères est d'environ $34000^{3}$. Par ailleurs, le nombre d'élèves étrangers inscrits dans des établissements japonais aux niveaux élémentaire et collège est de $64427^{4}$. Or, selon les statistiques portant sur les résidents étrangers, le nombre de mineurs étrangers en âge d'être scolarisés (de 6 à 15 ans) peut être estimé autour de $106000^{5}$. On peut donc en déduire qu'environ 41000 mineurs vivant au Japon ne sont pas scolarisés dans le système scolaire japonais ${ }^{6}$ et 7000 enfants risquent de nêtre inscrits dans aucun établissement scolaire public ou privé, japonais ou "étranger". Il faut toutefois

Le problème de la nonscolarisation des enfants

étrangers se pose aussi au niveau du lycée, dont l'entrée

est conditionnée par

un concours qui représente une barrière difficile à franchir

pour les élèves étrangers non japonophones. prendre ces chiffres avec précaution car le ministère de l'Éducation n'a pas pour mission de déterminer le nombre exact d'enfants non inscrits. Étant donné que l'obligation scolaire définie dans la loi fondamentale de l'éducation ne concerne que les personnes de nationalité japonaise, la non-scolarisation des enfants étrangers ne relève pas légalement de sa responsabilité. Le problème de la non-scolarisation des enfants étrangers se pose aussi au niveau du lycée, dont l'entrée est conditionnée par un concours qui repré- sente une barrière difficile à franchir pour les élèves étrangers non japonophones. Alors que $96 \%$ des élèves japonais accèdent au lycée et $57 \%$ à l'enseignement supérieur, les jeunes étrangers ont, par comparaison, un niveau de formation en moyenne inférieur, ce qui représente un lourd handicap sur le marché du travail.

Nous disposons, par ailleurs, des données établies par le ministère de l'Éducation concernant le nombre d'élèves ayant besoin d'un soutien en langue japonaise $e^{7}$. Ces données ne peuvent qu'offrir une idée approximative de la situation, dans la mesure où c'est aux enseignants de prendre la décision de demander une aide supplémentaire pour aider un élève à suivre les cours dans une classe ordinaire. Or ces demandes ne sont pas toutes satisfaites par les autorités locales. De plus, les chiffres sont difficiles à analyser dans la mesure où sont comptabilisés aussi bien les étrangers nouvellement arrivés que les Japonais naturalisés, ou encore les Japonais ayant accompli une partie de leur scolarité à l'étranger. C'est-à-dire que les demandes de la part des enseignants ne suffisent pas pour la mise en place de soutien en fonction des besoins urgents des élèves. En outre, certains élèves étrangers ou japonais (l'enquête du ministère de l'Éducation, de la Culture, des Sports, des Sciences et Technologies sur les demandes de soutien linguistique indique que 4895 cas concernent des élèves de nationalité japonaise) auraient aussi besoin d'un soutien pour suivre les cours, même s'ils maîtrisent la langue à l'oral et n'ont pas de difficultés sérieuses pour passer la journée dans une classe. Entre la compréhension d'une langue et sa maitrise suffisante pour comprendre, écrire, lire ou expliquer les concepts clés des différentes matières enseignées, nous savons bien qu'il existe plusieurs étapes. En 2008, le nombre d'élèves bénéficiant d'un soutien en japo- 
nais était de 19504 dans le primaire (3 791 établissements), 7576 au collège (2 028 établissements), et 1365 au lycée (342 établissements). Entre 1999 et 2008 , le chiffre total d'élèves bénéficiant de telles mesures a augmenté de $54 \%^{8}$. Des experts en la matière tels que Kôsei Sakuma et Haruo Ota soulignent qu'au-delà du manque de budget et d'enseignants de japonais comme seconde langue, le peu d'initiatives de la part du ministère de l'Éducation est notable ${ }^{9}$. D'après la même enquête, la question du soutien linguistique aux élèves étrangers concerne tous les départements sans exception, mais de façon très inégale. Par exemple, au niveau élémentaire, le département d'Aichi concentre 22,4\% des élèves soutenus (4 372 élèves), Shizuoka 11,6 \%, Kanagawa 9,1 \%, Tokyo 6,2 \%, Mie 5,8 \% et Osaka 3,7\%. Le manque de moyens ou d'enseignants est accru dans les départements, les villes ou les villages ${ }^{10}$ qui reçoivent peu d'élèves étrangers. Étant donné qu'au Japon les enseignants sont nommés par le département qui paye les deux tiers des salaires, le soutien scolaire pour les nouveaux arrivants est davantage négligé dans les départements où le nombre d'élèves étrangers est moindre. C'est également le cas dans les petites villes ou villages où, d'une part, les moyens budgétaires ne le permettent pas (les salaires des enseignants non titulaires sont pris en charge par les villes) et où, d'autre part, il est difficile de trouver des personnes ayant les compétences pour enseigner le japonais à des enfants étrangers ou des interprètes dans la langue maternelle de ces élèves.

Le problème de l'accès inégal à l'éducation se pose donc plus sérieusement dans les zones rurales que dans les grandes villes. Dans ces dernières, il y a, en effet, une concentration d'étrangers et un retour d'expérience de la part des anciens immigrés qui ont permis la création d'associations de soutien, la multiplication des liens avec l'administration locale et avec des personnes compétentes, expérimentées, voire professionnelles (interprètes dans les mairies, médiateurs scolaires ou enseignants de japonais expérimentés).

\section{La tendance assimilationniste de l'enseignement public}

La Constitution japonaise de 1946 n'accorde la jouissance du droit d'inscription dans les établissements scolaires qu'au peuple japonais : "kokumin" qui signifie littéralement "population du pays". S'il existe des débats contradictoires parmi les juristes pour savoir dans quelles mesures les étrangers sont concernés par la Constitution, l'interprétation la plus répandue dans la société est qu'ils ne le sont pas ${ }^{11}$. De ce fait, pour les étrangers, le droit à une vie décente (art. 25 de la Constitution) mais aussi les autres droits fondamentaux ne sont pas constitutionnellement

L'État ou les collectivités territoriales n'ont pas l'obligation d'assurer l'accès à l'enseignement scolaire aux résidents n'ayant pas la nationalité japonaise (art. 4) et les parents étrangers n'ont pas l'obligation de scolariser leurs enfants. (art. 5) reconnus ${ }^{12}$. Pour compen-

ser cette situation, l'article 2 des droits des collectivités locales accorde le droit à une assurance-vie, une couverture santé ou une aide sociale à tous les habitants. L'autonomie locale peut donc permettre de contrebalancer ce que la Constitution n'accorde pas aux non-Japonais, mais il faut savoir que cela dépend des départements. Ces derniers sont l'équivalent des conseils régionaux en France, le gouverneur y est élu au suffrage universel direct : certains sont très ouverts à l'internationalisation et à l'ac-

8. Ibid. 9. Voir Kôsei Sakuma (dir.), Gaikokujin no kodomo no fushugaku, op.cit. ; Haruo Ota, New comers no kodomo to nihon no gakkô bunka (Les Enfants des new comers et la Culture scolaire japonaise), Tokyo, Kokusaishoin, 2000. 10. Les demandes de soutien sont traitées soit par le département, soit par les communes si celles-ci comptent plus de 500 ooo habitants. 11. "Kokumin" est également parfois traduit par "nation", ce qui souligne encore la polysémie de ce terme et la difficulté de son interprétation dans le textes de droit (note des coordinateurs). 12. Comme la Constitution japonaise ne concerne que les Japonais, les étrangers n'ont pas de droits, mais normalement ils n'ont pas d'obligations non plus. La question est alors de savoir comment l'État et les collectivités locales justifient les obligations, par exemple celle de payer les impôts pour les résidents étrangers. En fait, dans chaque article de la Constitution parlant des droits et obligations, il est indiqué : “conformément aux décisions édictées par les lois". Le droit fiscal auquel fait référence l'article zo de la Constitution sur l'obligation de payer les impôts s'applique donc à "tout le monde, quelle que soit la nationalité" (Hiroshi Kaneko, Sozeihô (Le Droit fiscal), $13^{e}$ édition, Tokyo, Kôbunsha, 2008, p. 100). 
Mariko Saneto, 30 ans, vit à Tokyo depuis l'âge de 10 ans. Juriste d'origine philippine, elle s'investit au sein de l'association Kalabaw-no-kai en donnant des cours de japonais aux enfants et aux parents en situation irrégulière. () CAmille Millerand

cueil des étrangers, mais d'autres ne le sont pas et rechignent à protéger les résidents étrangers par des initiatives et par des lois votées par la collectivité locale. Ainsi, certaines collectivités locales refusent aux résidents en situation irrégulière tout accès aux services administratifs et sociaux et signalent systématiquement leur présence au Bureau d'immigration ou à la police.

La Constitution définit aussi, dans son article 26, le droit et l'obligation de l'inscription des enfants de 6 à 15 ans dans un établissement scolaire : "Le peuple (kokumin), conformément aux décisions édictées par les lois, a le droit de recevoir de façon équitable une éducation correspondant à ses capacités. Le peuple, conformément aux décisions édictées par les lois, doit permettre à tout enfant, fille ou garçon, sous sa responsabilité, de recevoir une éducation de base. L'éducation obligatoire est gratuite." La nouvelle loi fondamentale de l'éducation de 2006 (qui a inté- gralement remplacé celle de 1947) en prescrit ainsi les détails dans ses articles 1, 4 et $5^{13}$ : "Article 1 : L'objet de l'éducation. L'éducation vise au développement total de la personne, contribue à la formation de l'État et de la société pacifique, et forme un peuple en bonne santé et d'esprit indépendant." "Article 4 : Chances égales pour tout le peuple. L'éducation doit être accessible au peuple suivant les capacités individuelles de chacun sans qu'aucune discrimination soit établie d'après la race, les croyances, le sexe, la situation sociale ou la position économique de la famille." "Article 5 : L'obligation scolaire. Le peuple ayant des enfants sous sa garde a l'obligation de leur donner une éducation générale. (...) Dans les écoles sous leur responsabilité, l'État ou les collectivités territoriales ne doivent pas faire payer de frais de scolarité."

Les articles de loi et de la Constitution présentés ci-dessus permettent de ne pas inclure les étrangers dans la scolarité obligatoire suite à une définition restrictive du terme "peuple", compris comme étant exclusivement ceux qui ont la nationalité 
japonaise. Ce qui veut dire que l'État ou les collectivités territoriales n'ont pas l'obligation d'assurer l'accès à l'enseignement scolaire aux résidents n'ayant pas la nationalité japonaise (art. 4) et que les parents étrangers n'ont pas l'obligation de scolariser leurs enfants (art. 5).

\section{Pas de place pour les différences culturelles}

Prenons un exemple pour mieux comprendre ce que signifient ces articles dans la pratique. Les parents japonais ayant un enfant en âge scolaire reçoivent une fiche d'inscription de la part de la municipalité au moment de l'affectation scolaire, tandis que pour les enfants n'ayant pas la nationalité japonaise, cette démarche administrative n'est pas automatique : il faut faire une demande de permission d'inscription dans un établissement public. En aucun cas la municipalité n'a l'obligation d'inscrire un enfant non japonais, même si, en août 2003, le ministre de l'Administration générale, de l'Intérieur et des Postes et Télécommunications a publié un avis sur la scolarisation des enfants étrangers pour promouvoir leur inscription dans les établissements publics ${ }^{14}$. Dans un tel contexte, les politiques menées par les maires ou les gouverneurs sont donc décisives pour l'accueil des élèves étrangers.

Par ailleurs, une fois inscrit dans un établissement scolaire japonais, l'élève étranger est traité de la même façon que les Japonais. Ce principe d'égalité de traitement ne souffre aucune exception, même si le besoin se fait sentir d'en faire la demande pour les enfants étrangers. Cela remonte à l'histoire de la politique éducative menée par le gouvernement lors de l'invasion de la Corée, qui s'est en partie maintenue après la Seconde Guerre mondiale : une politique éducative marquée par le souci de formation au nationalisme au sens étroit du terme, c'est-à-dire d'assimilation à la culture et à la langue japonaises. La philosophie et l'orienta- tion de l'éducation au Japon restent influencées par cette idéologie d'un nationalisme fermé sur le peuple et sur la culture traditionnelle japonaise. Ce contexte explique les raisons pour lesquelles la création des écoles coréennes a été acceptée, mais en leur octroyant un statut précaire, dans la mesure où elles sont considérées comme d'un rang inférieur dans le classement du ministère de l'Éducation japonaise, et où leur certification n'est pas reconnue dans la société japonaise. En contrepartie, dans ces écoles, les élèves ont la possibilité de maintenir leur nom d'origine, d'hériter de la langue et de la culture de leurs parents.

De par cet héritage, les élèves étrangers qui s'inscrivent dans un établissement public ne peuvent espérer voir leur demande de droit à la différence culturelle, sociale ou ethnique écoutée. Dans la pratique, le contenu de l'enseignement doit être conçu dans le but de former le futur citoyen japonais. Cette éducation à orientation assimilationniste et nationaliste exclut la différence culturelle et la liberté de pensée et de convictions politiques ; elle est en contradiction avec l'article 4 de la loi fondamentale de l'éducation de
La philosophie et l'orientation de l'éducation au Japon restent influencées par cette idéologie d'un nationalisme fermé sur le peuple et sur la culture traditionnelle japonaise. ait ratifié les différents traités internationaux (Convention internationale délimination de toute forme de discriminations raciale, droit à l'apprentissage de l'Unesco, Recommandation concernant l'éducation pour la compréhension internationale, la coopération et la paix et l'éducation reliant les droits de l'homme et la liberté fondamentale de l'Unesco...), ni le souci d'une éducation respectant les différences culturelles, ni même le droit à l'éducation ne sont pris en compte. Les ratifications des traités ne suffisent pas et ne sont pas utilisées par la société civile ou par les milieux éducatifs afin de promouvoir l'ouverture du système éducatif aux non-Japonais ${ }^{15}$. 


\section{La discrimination des écoles coréennes}

Pour bien comprendre le problème des écoles ethniques, et particulièrement coréennes, au Japon, il faut savoir qu'il existe deux types d'écoles étrangères sur le territoire nippon : les écoles internationales et les écoles ethniques ou écoles de groupes nationaux ${ }^{16}$.

Les écoles internationales sont des établissements reconnus par les associations mondiales (Western Association of Schools \& Colleges, Association of Christian Schools International, European Council of International Schools). Elles sont 33 à avoir ce statut au Japon. Parmi les écoles ethniques, les plus nombreuses sont les écoles brésiliennes (95 établissements, dont une dizaine classées "autres établissements scolaires"). En deuxième place viennent les écoles coréennes (70), les autres comptant moins de cinq établissements : écoles chinoises, péruviennes, indiennes, françaises, allemandes, américaines, anglaises, russes, etc.

Ces établissements se concentrent pour l'essentiel dans la région de Tokyo (36\%), dans la région centrale très industrielle où se trouvent entre autres Nagoya et Shizuoka (35\%), ainsi que dans la région du Kansai (20\%). En outre, la loi fondamentale sur l'éducation (de 2006) et la loi sur l'enseignement scolaire de 1947 (dernier amendementen2011) distinguent deux catégories d'établissements scolaires. D'une part, les établissements qui se trouvent sous le contrôle du ministère de l'Éducation et des collectivités locales : on parle d'établissements de première catégorie (ichijôkôo $)^{17}$, et, d'autre part, les "autres établissements scolaires” (kakushu gakkôo) ${ }^{18}$, ce second groupe ne donnant pas accès à l'enseignement supérieur universitaire. Pour être reconnu par le ministère comme établissement de première catégorie, il faut satisfaire à différentes conditions, comme le contenu de l'enseignement, l'utilisation de manuels scolaires approuvés par le ministère, la qualification des enseignants et la propriété du terrain et du bâtiment par l'administrateur de l'établissement. L'intégration d'écoles étrangères dans cette catégorie s'est faite progressivement pour les écoles internationales, mais une discrimination particulière persiste à l'égard des écoles coréennes.

Les différences entre ichijôkô et kakushu gakkô sont grandes, non seulement au niveau financier mais aussi sur le plan des ressources humaines (emplois de bureau, infirmière, médecin scolaire, demipension, etc., qui sont obligatoires et payées par l'État et les collectivites locales pour les ichijôkô). Elles concernent également des aspects non négligeables de la vie quotidienne, comme la signalisation routière pour la sécurité des élèves aux abords des écoles, les réductions pour les transports en

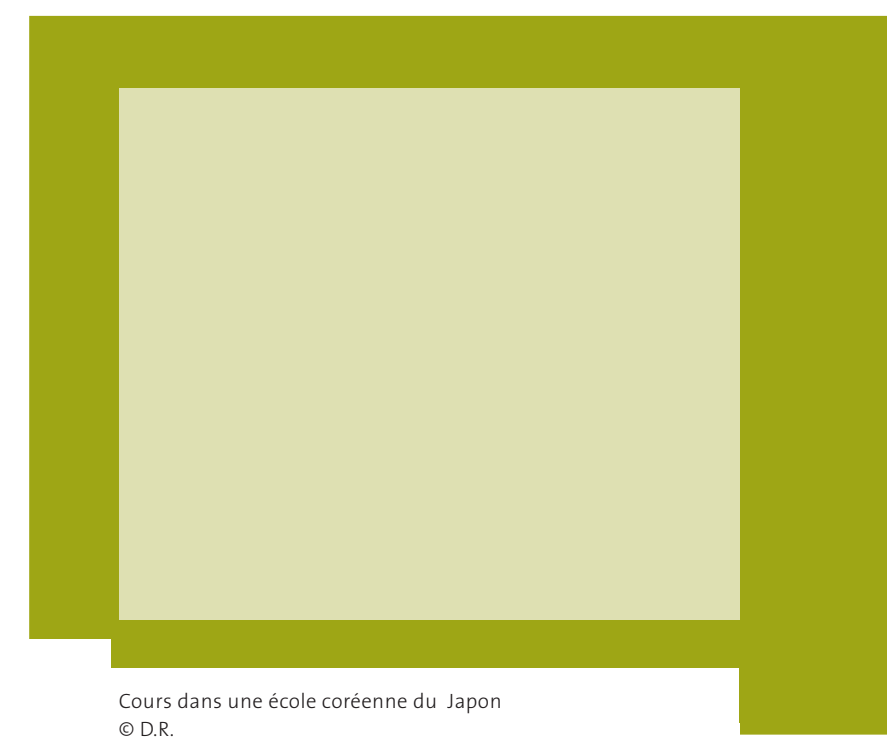

16. Seiji Fukusa et Mitsuko Suefuji (dir), Sekai no gaikokujin gakkô (Les Écoles étrangères dans le monde), Tokyo, Toshindo, 2005; Gekkan “lo" Henshubu (La rédaction du magazine mensuel IO), Nihon no nakano gaikokujingakko, (Les Écoles étrangères au Japon) Tokyo, Akashishoten, 2007. 17. Ichijôkô signifie "établissements scolaires définis par l’article 1 de la loi sur l'éducation". 18. Kakushu gakkô signifie "établissements scolaires autres que ichijôkô et senshû gakkô (écoles professionnelles d'une durée de plus d'un an)" définis par l'article 134 de la loi sur les établissements scolaires. 
commun (accordées aux kakushu gakkô en 1994), ou encore la participation au tournoi sportif des lycées (accordée en 1993).

Historiquement, les écoles coréennes sont les plus discriminées et les plus en difficulté sur les plans financier, politique et idéologique ${ }^{19}$. Le refus de soutenir, voire de reconnaître les écoles coréennes, en particulier les écoles des Coréens du Nord, est justifié par les liens qu'elles entretiennent avec l'Association générale des Coréens résidant au Japon (Sôren). En effet, cette association apporte également un important soutien politique et financier à la République populaire démocratique de Corée, or les relations entre le Japon et la Corée du Nord sont très tendues depuis la fin de la guerre de Corée et n’ont jamais été normalisées.

\section{Quand les pesanteurs de l'Histoire déterminent les choix éducatifs}

Un bref rappel historique est ici nécessaire. Parmi les 2 millions de Coréens qui avaient prévu de rentrer chez eux après la déclaration de Potsdam de 1945, près de 600000 sont restés au Japon. Dès janvier 1948, le ministère de l'Éducation déclara qu'il fallait inscrire tout enfant en âge d'être scolarisé dans les écoles japonaises car, à ce momentlà, ces enfants étaient encore des Japonais. De ce fait, les Coréens ont créé des centres de langue coréenne (kokugo kôshûjo), qui sont à l'origine des écoles coréennes actuelles. Ils les ont créées pour le maintien de leur langue et de leur culture d'origine en espérant retourner dans leur pays dans un avenir proche. Cependant, en avril 1952, une fois que le gouvernement japonais eut conclu le traité de paix de San Francisco, les Coréens perdirent leur nationalité japonaise. Ils devinrent des étrangers et n'eurent donc plus l'obligation de scolariser leurs enfants de 6 à 15 ans. Il faut noter qu'à cette époque, 95 \% des élèves étrangers étaient coréens, ce qui éclaire l'importance de ce problème jusquà aujourd'hui. En 1968, l'université coréenne (Chôsen daigakkô) de Tokyo, créée par les Coréens en 1954, a été la première à obtenir le statut d'“autre établissement scolaire" (kakushu gakkô), par permission du gouverneur de Tokyo. Malgré cela, la reconnaissance de ses diplômes reste limitée sur le marché du travail. Par contre, les étudiants peuvent s'inscrire au concours d'entrée des écoles doctorales des universités japonaises. En 2005,
Bien qu'elles accueillent le plus grand nombre d'élèves étrangers, les écoles coréennes continuent donc d'être fortement discriminées alors que les autorités pourraient, au contraire, en faire des écoles pilotes pour les autres établissements ethniques. le ministère de la Santé, de l'Emploi et de la Protection sociale accepte les candidatures au niveau de la licence de l'université coréenne de Tokyo pour les concours du personnel de la Sécurité sociale et de consultant fiscal.

En 1975, toutes les écoles coréennes sont reconnues comme "autres établissements scolaires" et obtiennent une subvention du département, ainsi que le droit de recevoir des dons exemptés d'impôts. Toutefois, cette dénomination "kakushu gakkô" n'autorise pas l'accès à l'enseignement supérieur, ni même la reconnaissance nationale du diplôme obtenu. Cette non-reconnaissance a longtemps discriminé les Coréens dans la société japonaise quand ils voulaient entrer dans un établissement scolaire japonais de première catégorie public ou privé. Ce n'est qu'en 1985 que 10 établissements coréens ont enfin été reconnus comme établissements de première catégorie.

On peut signaler trois autres formes de discrimination touchant tout particulièrement les écoles coréennes. Premièrement, la reconnaissance des qualifications de fin d'étude primaire, du collège ou du lycée délivrées par les "autres établissements scolaires” est encore limitée. Par contre, depuis 2003, 16 des 33 écoles internationales reconnues par les 
associations citées plus haut, ainsi que 40 établissements ethniques (brésiliens, chinois, français, allemands, etc.) ont obtenu l'autorisation pour leurs élèves de candidater aux concours d'entrée aux universités nationales, municipales ou privées. C'est-àdire que leur certification permet de s'inscrire aux concours, ce qui n'est pas le cas pour les 10 écoles coréennes reconnues de "première catégorie" (ichijôkô), dont les lycéens sont toujours soumis à un examen individuel afin que l'université évalue leurs compétences. Tout cela souligne combien les situations sont contrastées et la politique en la matière assez incohérente.

Deuxièmement, les 10 écoles coréennes de "première catégorie" ne sont pas autorisées à recevoir des dons exemptés d'impôts, contrairement aux écoles internationales et aux établissements japonais privés de "première catégorie". De ce fait, les établissements coréens, qui datent de plus d'un demi-siècle, ont souvent besoin de rénovation mais n'ont pas de moyens budgétaires suffisants. Enfin, un dernier débat en cours concerne la gratuité au niveau du lycée. En mars 2010, le gouvernement a adopté la loi de la gratuité des lycées publics et privés ${ }^{20}$, qu'ils soient de première catégorie ou classés "autres établissements scolaires". Malheureusement, au cours de la première année suivant l'adoption de la loi, le ministère de l'Éducation n'a appliqué cette décision qu'à une trentaine d'écoles internationales, à 8 établissements ethniques brésiliens et à d'autres établissements ethniques (péruviens, chinois, anglais, français, allemands, etc.) : les établissements coréens ont été exclus de cette mesure, sans que l'État donne la moindre raison ${ }^{21}$. Bien qu'elles accueillent le plus grand nombre d'élèves étrangers, les écoles coréennes continuent donc d'être fortement discriminées alors que les autorités pourraient, au contraire, en faire des écoles pilotes pour les autres établissements ethniques.

\section{Conclusion}

Les politiques d'éducation envers les élèves étrangers depuis la fin de la Seconde Guerre mondiale manquent de cohérence et sont parfois discriminantes, en particulier à l'égard des Coréens issus de la colonisation et installés au Japon depuis quatre générations. Malgré l'augmentation du nombre d'étrangers et la diversification culturelle de la population, le Japon a conservé la tendance assimilationniste de son système éducatif. Rappelons que, dans le passé, cette même tendance a déjà contribué à faire disparaître la langue et la culture des Aïnous $^{22}$. Une des demandes de la part des élèves étrangers porte sur la prise en compte de la diversité culturelle et linguistique dans l'enseignement public. Puisque la langue et la culture sont constitutives de l'identité, il est important, dans une société démocratique, que l'enseignement public joue aussi un rôle dans la transmission des langues et cultures d'origine (ou héritées) pour les personnes faisant partie des minorités ethniques. En attendant des évolutions au sein des écoles publiques, il pourrait être intéressant de favoriser les échanges entre les écoles ethniques et les établissements japonais du même quartier, ce qui assurerait à notre sens une meilleure connaissance des diversités culturelles, linguistiques ou religieuses. I

20. Les élèves des lycées publics devaient payer personnellement 118 ooo yens par an avant la loi sur la gratuité des lycées publics de 2010. Depuis, même pour les élèves ayant choisi des lycées privés, une subvention de 118 ooo yens sera versée à l'établissement par l'État. La famille doit néanmoins payer la différence, mais cette différence entre public et privé a diminué, sauf pour les élèves des écoles coréennes. Les explications fournies par le MEXT à cet égard ne sont pas claires. L'une est que certaines écoles coréennes reçoivent une subvention de la part de la Corée du Nord et que le commissaire aux comptes n'a pas le contrôle total de la circulation du budget. Mais il y a aussi les choix politiques des gouverneurs : quand le gouverneur n'est pas satisfait du programme scolaire (surtout en histoire et sciences politiques), il retire sa confiance à certaines écoles coréennes, qui dès lors ne reçoivent plus de subventions de la collectivité territoriale. Bien entendu, il faut ajouter que les tensions sont fortes entre la Corée du Nord et le Japon, surtout après le retour de 5 Japonais victimes d'un rapt coréen en 2002. 21. Plus précisément, deux établissements coréens ont bénéficié de cette mesure. Cependant, il ne s'agit pas d'écoles fréquentées par les Coréens du Japon, mais d'écoles reconnues par le ministère de l'Éducation de la Corée du Sud. Elles accueillent des élèves coréens arrivés récemment et qui envisagent de retourner en Corée du Sud. Voir MEXT, http://www.mext.go.jp/a_menu/shotou/mushouka/1307345.htm). 22. Les Aïnous sont un peuple autochtone qui vivait sur les îles au nord du Japon et qui a été combattu puis fortement discriminé par les représentants du pouvoir central. Ils ne sont aujourd'hui plus que 20 ooo environ et représentent une des principales voix s'opposant au discours de l'homogénéité ethnique du Japon (note des coordinateurs). 